\title{
Diguanylate Cyclases in Vibrio cholerae: Essential Regulators of Lifestyle Switching
}

\author{
Sumit Biswas*, Om Prakash Chouhan and Divya Bandekar \\ ViStA Lab, Department of Biological Sciences, Birla Institute of Technology and Sciences (BITS), Pilani-KK Birla Goa \\ Campus, Goa, India
}

Biofilm formation in Vibrio cholerae empowers the bacteria to lead a dual lifestyle and enhances its infectivity. While the formation and dispersal of the biofilm involves multiple components-both proteinaceous and non-proteinaceous, the key to the regulatory control lies with the ubiquitous secondary signaling molecule, cyclic-di-GMP (c-di-GMP). A number of different cellular components may interact with c-di-GMP, but the onus of synthesis of this molecule lies with a class of enzymes known as diguanylate cyclases (DGCs). DGC activity is generally associated with proteins possessing a GGDEF domain, ubiquitously present across all bacterial systems. V. cholerae is also endowed with multiple DGCs and information about some of them have been pouring in over the past decade. This review summarizes the DGCs confirmed till date in V. cholerae, and emphasizes the importance of DGCs and their product, c-di-GMP in the virulence and lifecycle of the bacteria.

Southern University of Science and

Technology, China

Reviewed by:

Umesh Ahuja,

UCLA Health System, United States

Badreddine Douzi,

INRA Centre Nancy-Lorraine, France

*Correspondence:

Sumit Biswas

sumit@goa.bits-pilani.ac.in

Specialty section:

This article was submitted to Molecular Bacterial Pathogenesis,

a section of the journal

Frontiers in Cellular and Infection

Microbiology

Received: 13 July 2020

Accepted: 14 September 2020

Published: 22 October 2020

Citation:

Biswas S, Chouhan OP and

Bandekar D (2020) Diguanylate

Cyclases in Vibrio cholerae: Essential

Regulators of Lifestyle Switching.

Front. Cell. Infect. Microbiol.

10:582947.

doi: $10.3389 /$ fcimb.2020.582947
Keywords: biofilm, GGDEF, cyclic-di-GMP, virulence, diguanylate cyclase

\section{INTRODUCTION}

\section{Vibrio cholerae: Dual Lifestyle and Biofilm}

Formation of biofilm enables the bacteria to survive and propagate despite the presence of antibiotics or other external stress. Vibrio cholerae is no exception. This bacterium adopts two different lifestyles-the motile pathogenic form in the human host, and the sessile form in waterbodies existing in associated biofilms. The biofilm allows the bacteria to survive nutrient limitations, fluctuations in oxygen levels, and massive changes in osmolarity (Rodney, 2002; Tischler and Camilli, 2004; Waters et al., 2008). Additionally, it also allows changes in the bacterial proteome by inducing favorable genes or suppressing unfavorable genes in order to adapt better.

Biofilm formation in $V$. cholerae is a three-step cyclic process, involving (a) surface attachment, (b) colony formation, and (c) dispersal. In the initial step (surface attachment), motile $V$. cholerae scan solid surfaces-with a preference for the chitinous exoskeleton of zooplanktons or phytoplanktons (Tamplin et al., 1990; Rawlings et al., 2007). The bacterium, powered by the single polar flagellum (with a $\mathrm{Na}^{+}$-driven motor and regulated by the Flh proteins) seeks a suitable surface (Echazarreta and Klose, 2019), and has been suggested to be quite selective in assaying the surface before selecting it for attachment (Utada et al., 2014). The Mannose-Sensitive Haemagglutinin type 4 surface pili (MSHA-pili) contribute to strong surface attachment during the initial attachment steps (Watnick et al., 1999; Wong, 2016).

After multiplication and the progression of colony formation, the size of the average member cell keeps on decreasing to increase the compaction in the biofilm. The size decreased from $2.4 \mu \mathrm{m}$ (Drescher et al., 2016) at the beginning of biofilm to $1.8 \mu \mathrm{m}$ for cellular communities 
having $\sim 1,000$ cells (Wong, 2016). Consequently, interbacterial distances in the biofilm matrix also show a significant decrease. The directionality of colony growth also changes incrementally with increase in colony size-while the initial growth is only one dimensional, growth happens in all three directions when cell number crosses 200. It is during this three-dimensional growth phase, the extracellular matrix composed of polysaccharides, proteins, and a small amount of nucleic acids (Joachim and Karl, 2002; Wong, 2016) is secreted. Vibrio polysaccharides (VPS) are essential for keeping the cells together and maintenance of the $3 \mathrm{D}$ structure. Proteins of the extracellular matrix, viz., RbmA, RbmC and Bap1 play critical roles in the biofilm as well. The RbmA protein has been implicated in cellular adhesion, architecture and biofilm stability process, while the RbmC secreted on the outer surface of the cells creates flexible scaffolds where the cells can grow and multiply. The Bap1 protein maintains pellicle strength and hydrophobicity allowing the biofilm to propagate at the water-air interface (Römling et al., 2013; Hay and Zhu, 2015).

The last phase of biofilm formation is the dispersal of the bacterial cells from the biofilm to search and colonize a new substratum when conditions are favorable. Environmental conditions such as high/low oxygen level, the concentration of phosphate, $\mathrm{Ca}^{2+}$, etc, have negative effects (inhibition of $v p s$ gene transcription) on biofilm formation and induce the dispersal of the V. cholerae biofilm (Colwell and Huq, 1994; Hay and Zhu, 2015). Atleast two deoxyribonucleases and the Xds protein have also been reported to play crucial roles in biofilm dispersal (Römling et al., 2013; Sisti et al., 2013). The degradation of biofilm and extracellular matrix is induced by various environmental signals and other proteins, many of which are yet to be elucidated.

\section{REGULATION OF BIOFILM FORMATION IN V. CHOLERAE AND PATHOGENESIS}

Formation of the biofilm comes at a premium - the amount of resources diverted and spent toward the formation is substantial, but the benefits are huge. Being able to thrive in adverse conditions accords the bacterium a different strategy for survival. Therefore, the process needs to be highly regulated and that is how it happens, with the interplay of various factors. In $V$. cholerae, transcriptional activators, repressor proteins and sigma factors RpoS and RpoE have been demonstrably involved in the process (He et al., 2012).

The structural genes for VPS synthesis have been reported to be essential for exopolysaccharide biosynthesis and biofilm formation (Yildiz and Schoolnik, 1999). These genes, located on $v p s-1$ ( $v p s A$ to $v p s K)$ and $v p s-2$ ( $v p s L$ to $v p s Q)$ operons, are positively regulated by $\mathrm{VpsR}$ and $\mathrm{VpsT}$, while HapR negatively regulates the expression of the vps genes, and the positive regulators VpsR and VpsT themselves (Casper-Lindley and Yildiz, 2004; Beyhan et al., 2007). Both VpsR and VpsT bind directly to the $v p s$ promoter regions and have recognition sites in $v p s-1, v p s-2$ and $v p s-L$ operons which act as regulatory sequences in the expression of extracellular polysaccharide and matrix protein synthesis (Fong et al., 2010). A recent report relates the activation of the $v p s$ operons to the concentration of $\mathrm{VpsR}$ as well as c-di-GMP (Hsieh et al., 2020) directly affecting the o70 RNAP. Additionally, VpsT can act as a regulatory protein with recognition sequences for $\mathrm{RbmA}$, whereas $\mathrm{RbmC}$ and Bap1 promoters also contain recognition sites for VpsR (Boyd and O’Toole, 2012; Zhao-Xun, 2015).

Activation of HapR is an important precursor to the process of biofilm dispersion. The N-terminal HTH domain of HapR directly binds to the $v p s-2$ operon at $v p s L$ and $v p s T$ (Jonas et al., 2008; Sudarsan et al., 2008). The activation of HapR is controlled by small molecules involved in the quorum sensing pathway. During the biofilm phase, lower concentrations of the quorum sensing molecules AI-2 and CAI-1 activate the transcription of quorum sensing regulatory RNAs (sRNA, via phosphorylation of RpoN and LuxO), which prevent the synthesis of HapR. With the increase in concentrations of AI-2 and CAI-1, LuxO is dephosphorylated, and the sRNAs are repressed, leading to the expression of HapR, eventually resulting in the dispersal of the biofilm (Tchigvintsev et al., 2010). Other negative regulators include the cAMP and the cAMP-receptor protein complex (Liang et al., 2007).

Intricately involved with all these regulatory elements, including those involved in pathogenesis is the secondary signaling messenger molecule cyclic-di-GMP (Figure 1; Watnick and Kolter, 2000; Tischler and Camilli, 2005). Both the biofilm activators, VpsT and VpsR can bind to c-di-GMP and has been shown to be responsive to fluctuations in the intracellular concentrations of c-di-GMP in V. cholerae (Krasteva et al., 2012; Hay and Zhu, 2015). An increase in the cellular c-di-GMP pool leads to the dimerization and activation of VpsT to induce biofilm formation (Shikuma et al., 2012). Similarly, allosteric activation of VpsR happens when the intracellular concentration of c-diGMP rises. The activation of both VpsR and VpsT enhances the expression of genes essential for the formation of the biofilm. The third major component which responds to changes in c-diGMP concentration is the $\sigma^{54}$-dependent activator FlrA, which is linked to the expression of flagellar motility. Increased c-diGMP levels lead to binding of c-di-GMP to FlrA, and inhibition of its activity which in turn diminishes flagellar gene expression (Srivastava et al., 2013). The dynamic extension and retraction of the MSHA pilus (Jones et al., 2015; Wang et al., 2016) is regulated by c-di-GMP via interaction with the ATPase MshE (Floyd et al., 2020). The role of c-di-GMP in the regulation of large adhesins which control reversible cell attachment during biofilm formation also highlights the essentiality of the molecule (Kitts et al., 2019). It is safe to state that c-di-GMP is a crucial and essential regulatory element for surface attachment and biofilm formation in $V$. cholerae.

Biofilm formation would therefore, be ideally associated with the loss of motility and switch toward the sessile, non-pathogenic lifestyle. However, the formation of biofilm is not just an essential ability which enhances the infectivity of $V$. cholerae (ZamoranoSánchez et al., 2019), but also has been found to be crucial to the process of intestinal colonization. Interestingly, $\mathrm{Xu}$ et al. (2003) found that the expression of biofilm genes (vpsA and $\operatorname{rbmA}$ ) was higher in rabbit ileal loop models. However, other biofilm-promoting genes like the $\mathrm{rbmC}$ and bap1 did not seem to have any role to play in intestinal infection models (Fong 


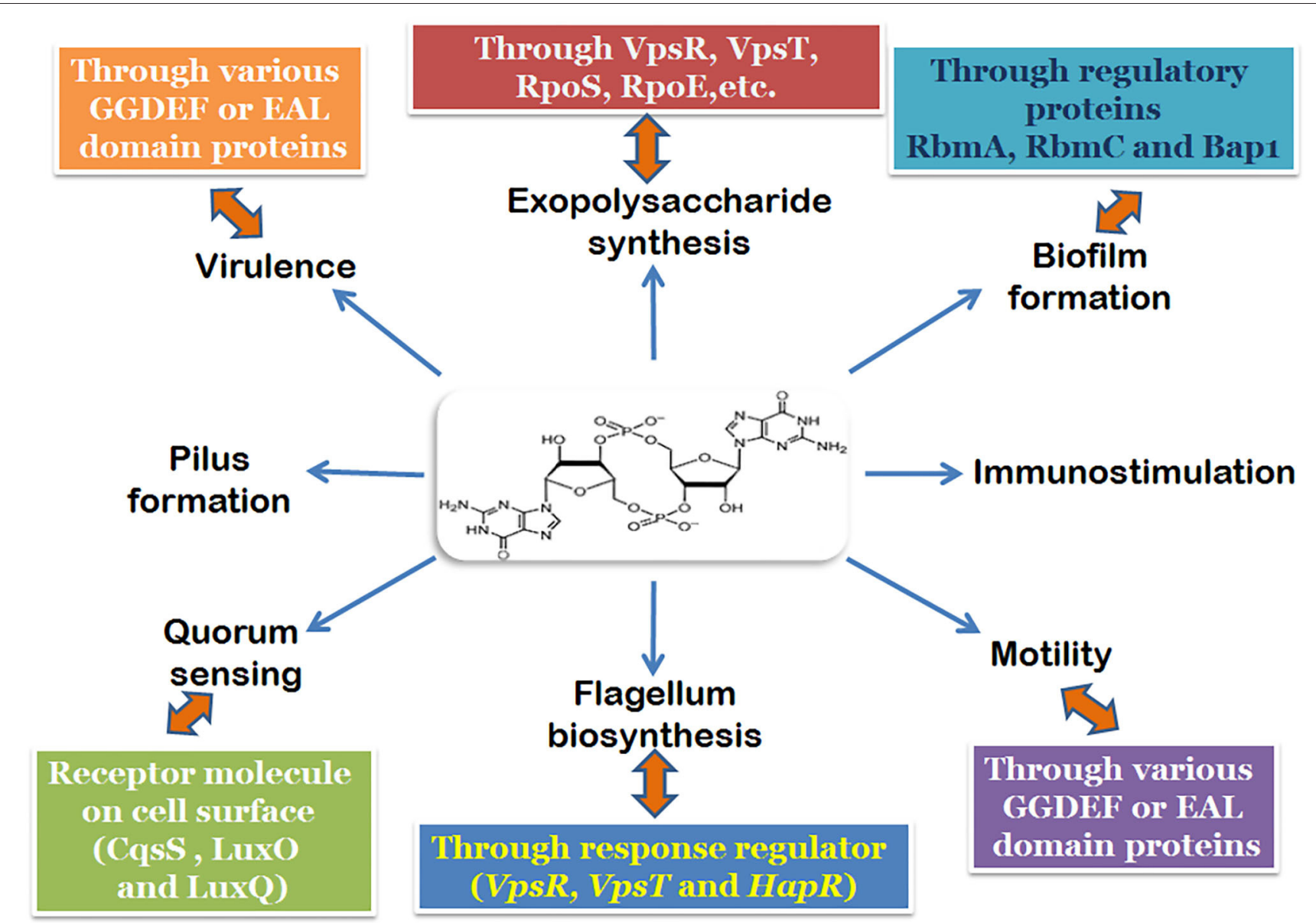

FIGURE 1 | Regulatory pathways and components of the biofilm formation mechanism involving c-di-GMP in $V$. cholerae.

et al., 2006), suggesting that the biofilms formed during intestinal colonization do not proceed beyond the RbmA-dependent primary cell aggregates (Silva and Benitez, 2016). Once in the intestine, the bacterium is exposed to multiple reverses like the effect of taurocholate salts in bile (Hay and Zhu, 2015) which is believed to degrade the VPS of the biofilm. Further repression of $v p s$ expression happens when the mucus layer is encountered, and the subsequent dispersal of the biofilm (Liu et al., 2007) results in the faster movement of the released bacterium in mucus. It has been postulated that the components of mucin might repress $v p s$ expression by actually regulating intracellular c-di-GMP concentrations (Liu et al., 2015) during V. cholerae infection. However, there has been no further elucidation of the interactions between mucin and c-di-GMP to explain the possible switch in the intestine.

\section{c-di-GMP AND DIGUANYLATE CYCLASES IN V. CHOLERAE}

Cellular c-di-GMP levels are regulated by the synthesis of activities of c-di-GMP by diguanylate cyclases (DGCs), and degraded by phosphodiesterases (Römling et al., 2013; Bandekar et al., 2017). Apart from Mycobacterium smegmatis (only two DGCs) (Kumar and Chatterjee, 2008), there is an abundance of DGCs in different bacterial systems (Römling et al., 2013; Chouhan et al., 2016). The multitude of functionalities regulated by DGCs and phosphodiesterases is very wide and even after years of investigation, the roles that they execute in these processes are not fully understood. The consensus is that the competitive action of the DGCs (and even the phosphodiesterases) results in the complex interactions between various pathways, but how or why these happen is yet to be elucidated. Even the response of DGCs to various extracellular signals and quorum sensing involves an intricate, networkmodulated pathway, which might need years to unravel.

In $V$. cholerae, sensing environmental cues in the surrounding water or in the small intestine have been closely associated with fluctuations in the intracellular c-di-GMP pool. Generally, an increase in the levels of cellular c-di-GMP is associated with the suppression of the virulence genes in $V$. cholerae (Tischler and Camilli, 2005; Tamayo et al., 2007). Currently, it is accepted that the bacterium invades the gastrointestinal (GI) cavity with augmented levels of c-di-GMP, which are acted 

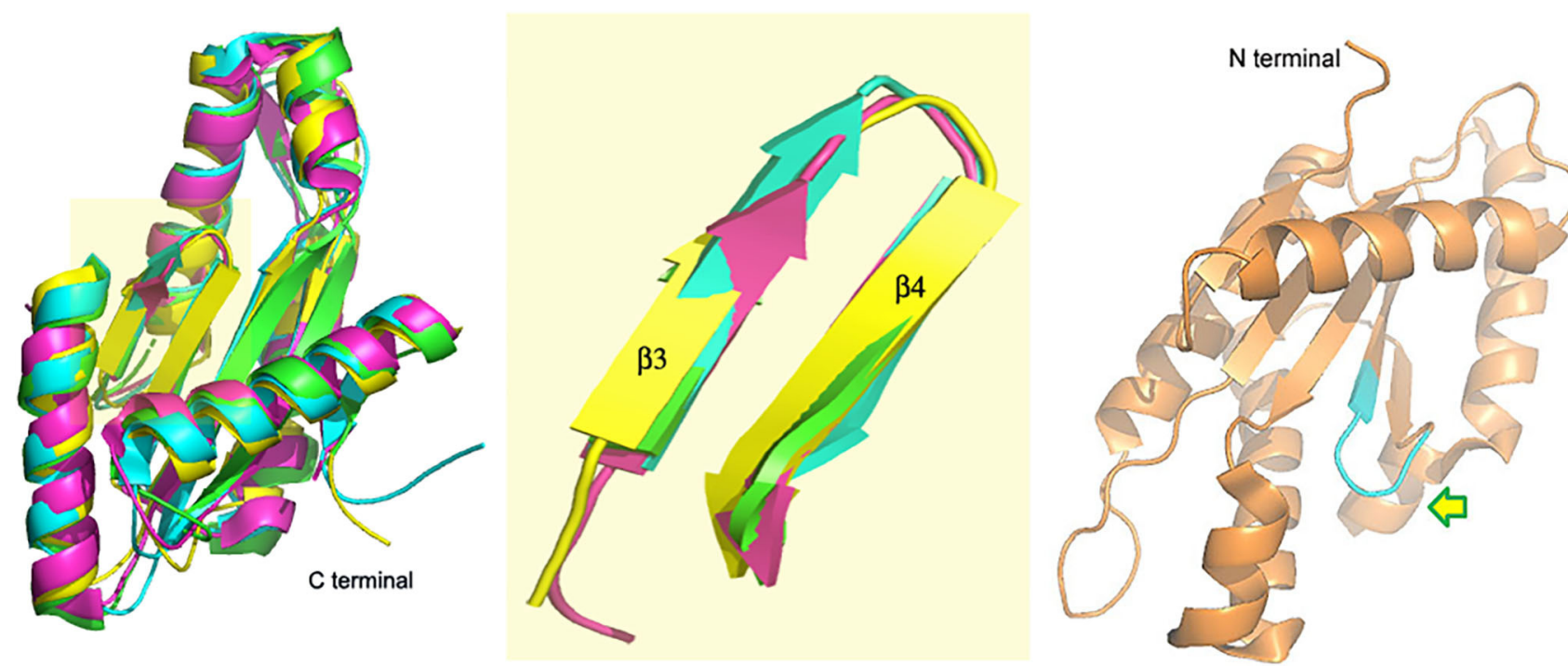

FIGURE 2 | VC0395_0300(161-321) (yellow, 6EIB) aligned with SadC (blue, 4WXW, Pseudomonas aeruginosa) and DGCs from Thermotoga maritima (green, 4URG) and Bdellovibrio bacteriovorus (pink, 6HBZ) Highlight: Alignment of GGDEF domains. Right: Active site of VC0395_0300 in cyan. Arrow shows direction of entry of GTP.

upon by the mucin components and eventually, the action of the phosphodiesterases bring down the c-di-GMP concentration (Koestler and Waters, 2014). During the late infection phase, though, there have been reports of a spurt in c-di-GMP concentration with expression of DGCs (Tamayo et al., 2007). The fluorescent visualization of the distribution of vibrios in the small intestine (Millet et al., 2014) has also brought to light the differential localizations in distinct niches along the small intestine, limited by the abundance of mucin. Together, these cues point to the following scenario- $V$. cholerae invades the GI tract with high levels of cellular c-di-GMP, which is brought down subsequently during the infective stage of the lifecycle. Once the bacteria is in the distal parts of the small intestine, where mucus is less abundant, c-di-GMP levels are raised again, as if in preparation for the life upon exit from the human host.

\section{Diguanylate Cyclases of $\boldsymbol{V}$. cholerae}

Diguanylate cyclases, responsible for c-di-GMP synthesis in bacteria, have been associated with a conserved GGD(/E)EF motif across different families (Ryjenkov et al., 2005). In line with the multiplicity of these proteins in bacteria, $V$. cholerae has been known to have 31 different proteins with a conserved GGD(/E)EF domain and 10 with a GGD(/E)EF and EAL (phosphodiesterase) domain in tandem distributed across its two chromosomes (https://www.ncbi.nlm.nih.gov/Complete_ Genomes/c-di-GMP.html) (Conner et al., 2017). However, not all of these are associated with motility and/or biofilm formation, and some have not been demonstrated to have DGC activity. Generally DGCs have an active site (A site) where the synthesis of c-di-GMP takes place and a site for allosteric control (RXXD) which regulates the synthesis. We would elaborate on the few DGCs from $V$. cholerae which have been elucidated over the years.

\section{CdgD}

When the GGDEF domain was still named as a domain of unknown function (DUF), Yildiz et al. (2004) had identified five genes encoding proteins with GGDEF and GGDEF plus EAL domains which were differentially expressed between the smooth and rugose variants of $V$. cholerae. The proteins encoded by these genes were named $\mathrm{Cdg} \mathrm{A}-\mathrm{E}$ and assayed for their expression. Of these, the $\mathrm{CdgD}$ and $\mathrm{CdgC}$ deletion mutants showed significant alteration in the biofilm formation of the strains harboring them. While CdgD had a GGDEF domain along with a sensory PAS domain, CdE showed the presence of both GGDEF and EAL domains (Lim et al., 2006). While deletion of $c d g D$ caused an increase in motility, $c d g C$ mutants were associated with a 2.3-fold decrease in motility. $\mathrm{CdgD}$ was later characterized as a diguanylate cyclase and $\mathrm{CdgC}$ was responsible for negative regulation of VPS biosynthesis (Lim et al., 2007).

\section{$\mathrm{CdgH}$}

Subsequently, (Beyhan et al., 2007) reported the activity of another protein with a predicted GGDEF domain, which they named $\mathrm{CdgH}$. Overexpression of $c d g H$ resulted in a high amount of c-di-GMP accumulation in the cell, which established $\mathrm{CdgH}$ as a diguanylate cyclase. Additionally, $\mathrm{CdgH}$ positively regulated the rugosity of the cell. The structure of $\mathrm{CdgH}$ is one of the two solved $V$. cholerae DGC structures, and displayed the presence of two Nterminal tandem periplasmic substrate-binding ( $\mathrm{PBPb})$ domains for signal recognition ( $\mathrm{Xu}$ et al., 2017). Additionally, the same group had characterized several other predicted GGDEF domain proteins, which were not however DGCs.

\section{VCA0965}

A further DGC in $V$. cholerae was reported by the Waters lab in 2014 (Hunter et al., 2014). Interestingly, this protein did 
not have the conserved GGDEF motif, but had a degenerate AGDEF site. Significantly, expression of VCA0965 in V. cholerae was shown to cause a three-fold reduction in flagellar-based motility. This was noteworthy as many of the other predicted GGDEF proteins with conserved sequence did not show DGC activity, whereas VCA0965, despite its degenerate active site, could synthesize c-di-GMP.

\section{VC0395_0300}

A DGC with a GGEEF domain was reported by our group in 2017 (Bandekar et al., 2017; Chouhan and Biswas, 2018). While VC0395_0300 was shown to synthesize c-di-GMP actively and had an essential role to play in the biofilm formation of $V$. cholerae, mutations at the central positions of the GGEEF sequence were detrimental to the functional activity of the protein (Chouhan et al., 2016). The structure of the protein though showed similar architecture (Figure 2) associated with diguanylate cyclases from other bacterial systems (Chouhan et al., 2020). Another deviation in this DGC was that it lacked the site for allosteric inhibition found in the other DGCs of $V$. cholerae, suggesting a different mode of inhibitory control in this DGC.

\section{CONCLUDING REMARKS}

The secondary messenger c-di-GMP plays the most crucial role in the regulation of biofilm formation and motility of $V$. cholerae. The levels of intracellular c-di-GMP are modulated by a host of factors including the diguanylate cyclases from which these are synthesized, the phosphodiesterases which lead to their degradation, and other receptor molecules including several virulence genes. The abundance of GGDEF domains in bacterial species, coupled with the uncertainty around their

\section{REFERENCES}

Bandekar, D., Chouhan, O. P., Mohapatra, S., Hazra, M., Hazra, S., and Biswas, S. (2017). Putative protein VC0395_0300 from Vibrio cholerae is a diguanylate cyclase with a role in biofilm formation. Microbiol. Res. 202, 61-70. doi: 10.1016/j.micres.2017.05.003

Beyhan, S., Bilecen, K., Salama, S. R., Casper-Lindley, C., and Yildiz, F. H. (2007). Regulation of rugosity and biofilm formation in Vibrio cholerae: comparison of VpsT and VpsR regulons and epistasis analysis of vpsT, vpsR, and hapR. J. Bacteriol. 189, 388-402. doi: 10.1128/JB.00981-06

Boyd, C. D., and O'Toole, G. A. (2012). Second messenger regulation of biofilm formation: breakthroughs in understanding c-di-GMP effector systems. Annu. Rev. Cell Dev. Biol. 28, 439-462. doi: 10.1146/annurev-cellbio-101011-155705

Burdette, D. L., Monroe, K. M., Sotelo-Troha, K., Iwig, J. S., Eckert, B., Hyodo, M., et al. (2011). STING is a direct innate immune sensor of cyclic di-GMP. Nature 478, 515-518. doi: 10.1038/nature 10429

Casper-Lindley, C., and Yildiz, F. H. (2004). VpsT is a transcriptional regulator required for expression of vps biosynthesis genes and the development of rugose colonial morphology in Vibrio cholerae O1 El Tor. J. Bacteriol. 186, 1574-1578. doi: 10.1128/JB.186.5.1574-1578.2004

Chouhan, O. P., Bandekar, D., Hazra, M., Baghudana, A., Hazra, S., and Biswas, S. (2016). Effect of site-directed mutagenesis at the GGEEF do- main of the biofilm forming GGEEF protein from Vibrio cholerae. AMB Express 6:2. doi: 10.1186/s13568-015-0168-6

Chouhan, O. P., and Biswas, S. (2018). Subtle changes due to mutations in the GGDEF domain result in loss of biofilm forming activity in the VC0395_0300 function as diguanylate cyclases renders further complexity to the mechanism of action of this class of enzymes. To add to the conundrum, the ability of degenerate GGDEF domains to synthesize c-di-GMP and the variance of allosteric inhibitory mechanisms in the DGCs are also systems of interest. It has been hypothesized that the multiple DGCs don't fire in unison-one or a few of them might be expressed at a time, possibly in response to an environmental cue. The association of the DGCs with an extra sensory domain in most cases points to the interaction of the DGC with the extracellular environment. Elucidation of the modes of action of the other DGCs and their regulation vis-à-vis the sensory domain will lead to solving the enigma of multiplicity of the DGCs.

The hitherto unexplored role of c-di-GMP against the host immune system is also an area of intrigue which has been poorly explored. In mammals, c-di-GMP was found to activate the innate immune system by binding to STING (stimulator of interferon genes) (Burdette et al., 2011). However, how the host immune response affects the levels of intercellular c-di-GMP also needs to be explored and should open up newer areas of understanding of this signaling messenger. The observation of hyperinfectivity (a short-lived but elevated infectious state where the virulence gene expression is high) in biofilm-grown cells of $V$. cholerae in comparison to planktonic cells (Gallego-Hernandez et al., 2020), makes it extremely important to understand the mechanism of biofilm-formation in the bacteria.

\section{AUTHOR CONTRIBUTIONS}

$\mathrm{SB}, \mathrm{OC}$, and $\mathrm{DB}$ contributed to the drafting and writing of the manuscript. All authors contributed to the article and approved the submitted version. protein from Vibrio cholerae, but no major change in the overall structure. Protein Pept. Lett. 25, 740-747. doi: 10.2174/0929866525666180628162405

Chouhan, O. P., Roske, Y., Heinemann, U., and Biswas, S. (2020). Structure of the active GGEEF domain of a diguanylate cyclase from Vibrio cholerae. Biochem. Biophys. Res.Commun. 523, 87-292. doi: 10.1016/j.bbrc.2019.11.179

Colwell, R. R., and Huq, A. (1994). Environmental reservoir of Vibrio cholerae. the causative agent of cholera. Ann. N. Y. Acad. Sci. 740, 44-54. doi: 10.1111/j.1749-6632.1994.tb19852.x

Conner, J. G., Zamorano-Sánchez, D., Park, J. H., Sondermann, H., and Yildiz, F. H. (2017). The ins and outs of cyclic di-GMP signaling in Vibrio cholerae. Curr. Opin. Microbiol. 36, 20-29. doi: 10.1016/j.mib.2017.01.002

Drescher, K., Jörn, D., Carey, D. N., Sven van, T., Ivan, G., Ned, S. W., et al. (2016) Architectural transitions in Vibrio cholerae biofilms at single-cell resolution. Proc. Natl. Acad. Sci. U.S.A. 113, E2066-E2072. doi: 10.1073/pnas.1601702113

Echazarreta, M. A., and Klose, K. E. (2019). Vibrio flagellar synthesis. Front. Cell. Infect. Microbiol. 9:131. doi: 10.3389/fcimb.2019.00131

Floyd, K. A., Lee, C. K., Xian, W., Nametalla, M., Valentine, A., Crair, B., et al. (2020). c-di-GMP modulates type IV MSHA pilus retraction and surface attachment in Vibrio cholerae. Nat. Commun. 11:1549. doi: 10.1038/s41467-020-15331-8

Fong, J. C., Karplus, K., Schoolnik, G. K., and Yildiz, F. H. (2006). Identification and characterization of $\mathrm{RbmA}$, a novel protein required for the development of rugose colony morphology and biofilm structure in Vibrio cholerae. J. Bacteriol. 188, 1049-1059. doi: 10.1128/JB.188.3.1049-1059.2006

Fong, J. C., Syed, K. A., Klose, K. E., and Yildiz, F. H. (2010). Role of Vibrio polysaccharide (vps) genes in VPS production, biofilm 
formation and Vibrio cholerae pathogenesis. Microbiology 156, 2757-2765. doi: 10.1099/mic.0.040196-0

Gallego-Hernandez, A. L., DePas, W. H., Park, J. H., Teschler, J. K., Hartmann, R., Jeckel, H., et al. (2020). Upregulation of virulence genes promotes Vibrio cholerae biofilm hyperinfectivity. Proc. Natl. Acad. Sci. U.S.A. 117, 11010-11017. doi: 10.1073/pnas.1916571117

Hay, A. J., and Zhu, J. (2015). Host intestinal signal-promoted biofilm dispersal induces Vibrio cholerae colonization. Infect. Immun. 83, 317-323. doi: 10.1128/IAI.02617-14

He, H., Cooper, J. N., Mishra, A., and Raskin, D. M. (2012). Stringent response regulation of biofilm formation in Vibrio cholerae. J. Bacteriol. 194, 2962-2972. doi: 10.1128/JB.00014-12

Hsieh, M. L., Waters, C. M., and Hinton, D. M. (2020). VpsR directly activates transcription of multiple biofilm genes in Vibrio cholerae. J. Bacteriol. 202, e00234-e00220. doi: 10.1128/JB.00234-20

Hunter, J. L., Severin, G. B., Koestler, B. J., and Waters, C. M. (2014). The Vibrio cholerae diguanylate cyclase VCA0965 has an AGDEF active site and synthesizes cyclic di-GMP. BMC Microbiol. 14:22. doi: 10.1186/1471-2180-14-22

Joachim, R., and Karl, E. K. (2002). Vibrio cholerae and cholera: out of the water and into the host. FEMS Microb. Rev. 26, 125-139. doi: 10.1111/j.1574-6976.2002.tb00605.x

Jonas, K., Edwards, A. N., Simm, R., Romeo, T., Römling, U., and Melefors, O. (2008). The RNA binding protein CsrA controls cyclic di-GMP metabolism by directly regulating the expression of GGDEF proteins. Mol. Microbiol. 70, 236-257. doi: 10.1111/j.1365-2958.2008.06411.x

Jones, C. J., Utada, A., Davis, K. R., Thongsomboon, W., Zamorano-Sánchez, D., Banakar, V., et al. (2015). c-di-GMP regulates motile to sessile transition by modulating MshA pili biogenesis and near-surface motility behavior in Vibrio cholerae. PLoS Pathog 11:e1005068. doi: 10.1371/journal.ppat.1005068

Kitts, G., Giglio, K. M., Zamorano-Sánchez, D., Park, J. H., Townsley, L., Cooley, R. B., et al. (2019). A conserved regulatory circuit controls large adhesins in Vibrio cholerae. mBio 10, e02822-19. doi: 10.1128/mBio.02822-19

Koestler, B. J., and Waters, C. M. (2014). Bile acids and bicarbonate inversely regulate intracellular cyclic di-GMP in Vibrio cholerae. Infect. Immun. 82, 3002-3014. doi: 10.1128/IAI.01664-14

Krasteva, P. V., Giglio, K. M., and Sondermann, H. (2012). Sensing the messenger: the diverse ways that bacteria signal through c-di-GMP. Protein Sci. 21, 929-948. doi: 10.1002/pro.2093

Kumar, M., and Chatterjee, D. (2008). Cyclic di-GMP: a second messenger required for long-term survival, but not for biofilm formation in Mycobacterium smegmatis. Microbiology 154, 2942-2955. doi: 10.1099/mic.0.2008/017806-0

Liang, W., Silva, A. J., and Benitez, J. A. (2007). The cyclic AMP receptor protein modulates colonial morphology in Vibrio cholerae. Appl. Environ. Microbiol. 73, 737482-737487. doi: 10.1128/AEM.01564-07

Lim, B., Beyhan, S., Meir, J., and Yildiz, F. H. (2006). Cyclic-diGMP signal transduction systems in Vibrio cholerae: modulation of rugosity and biofilm formation. Mol. Microbiol. 60, 331-348. doi: 10.1111/j.1365-2958.2006. 05106.x

Lim, B., Beyhan, S., and Yildiz, F. H. (2007). Regulation of Vibrio polysaccharide synthesis and virulence factor production by CdgC a GGDEF-EAL domain protein in Vibrio cholerae. J. Bacteriol. 189, 717-729. doi: 10.1128/JB. 00834-06

Liu, Z., Stirling, F. R., and Zhu, J. (2007). Temporal quorum-sensing induction regulates Vibrio cholerae biofilm architecture. Infect. Immun. 75, 122-126. doi: 10.1128/IAI.01190-06

Liu, Z., Wang, Y., Liu, S., Sheng, Y., Rueggeberg, K. G., Wang, H., et al. (2015). Vibrio cholerae represses polysaccharide synthesis to promote motility in mucosa. Infect. Immun. 83, 1114-1121. doi: 10.1128/IAI.02841-14

Millet, Y. A., Alvarez, D., Ringgaard, S., von Andrian, U. H., Davis, B. M., and Waldor, M. K. (2014). Insights into Vibrio cholerae intestinal colonization from monitoring fluorescently labeled bacteria. PLoS Pathog. 10:e1004405. doi: 10.1371/journal.ppat.1004405

Rawlings, T. K., Ruiz, G. M., and Colwell, R. R. (2007). Association of Vibrio cholerae O1 El Tor and O139 Bengal with the copepods acartia tonsa and Eurytemora affinis. Appl. Environ. Microbiol. 73, 7926-7933. doi: 10.1128/AEM.01238-07
Rodney, M. D. (2002). Biofilms: microbial life on surfaces. Emerg. Infect. Dis. 8, 881-890. doi: 10.3201/eid0809.020063

Römling, U., Galperin, M. Y., and Gomelsky, M. (2013). Cyclic di GMP: the first 25 years of a universal bacterial second messenger. Microbiol. Mol. Biol. Rev. 77, 1-52. doi: 10.1128/MMBR.00043-12

Ryjenkov, D. A., Tarutina, M., Moskvin, O. V., and Gomelsky, M. (2005). Cyclic diguanylate is a ubiquitous signaling molecule in bacteria: insights into biochemistry of the GGDEF protein domain. J. Bacteriol. 187, 1792-1798. doi: 10.1128/JB.187.5.1792-1798.2005

Shikuma, N. J., Fong, J. C. N., and Yildiz, F. H. (2012). Cellular levels and binding of c-di-GMP control subcellular localization and activity of the Vibrio cholerae transcriptional regulator VpsT. PLoS Pathog. 8: e1002719. doi: 10.1371/journal.ppat.1002719

Silva, A. J., and Benitez, J. A. (2016). Vibrio cholerae biofilms and cholera pathogenesis. PLoS Negl. Trop. Dis. 10:e0004330. doi: 10.1371/journal.pntd.0004330

Sisti, F., Ha, D. G., O’Toole, G. A., Hozbor, D., and Fernández, J. (2013). Cyclic-di-GMP signalling regulates motility and biofilm formation in Bordetella bronchiseptica. Microbiology 159, 869-879. doi: 10.1099/mic.0.06 4345-0

Srivastava, D., Hsieh, M. L., Khataokar, A., Neiditch, M. B., and Waters, C. M. (2013). Cyclic di-GMP inhibits Vibrio cholerae motility by repressing induction of transcription and inducing extracellular polysaccharide production. Mol. Microbiol. 90, 1262-1276. doi: 10.1111/mmi.12432

Sudarsan, N., Lee, E. R., Weinberg, Z., Moy, R. H., Kim, J. N., Link, K. H., et al. (2008). Riboswitches in eubacteria sense the second messenger cyclic di-GMP. Science 321, 411-413. doi: 10.1126/science.1159519

Tamayo, R., Pratt, J. T., and Camilli, A. (2007). Roles of cyclic diguanylate in the regulation of bacterial pathogenesis. Annu. Rev. Microbiol. 61, 131-148. doi: 10.1146/annurev.micro.61.080706.093426

Tamplin, M. L., Gauzens, A. L., Huq, A., Sack, D. A., and Colwell, R. R. (1990). Attachment of Vibrio cholerae serogroup O1 to zooplankton and phytoplankton of Bangladesh waters. Appl. Env. Microbiol. 56, 1977-1980. doi: 10.1128/AEM.56.6.1977-1980.1990

Tchigvintsev, A., Xu, X., Singer, A., Chang, C., Brown, G., Proudfoot, M., et al. (2010). Structural insight into the mechanism of c-di-GMP hydrolysis by EAL domain phosphodiesterases. J. Mol. Biol. 402, 524-538. doi: 10.1016/j.jmb.2010.07.050

Tischler, A. D., and Camilli, A. (2004). Cyclic diguanylate (c-di-GMP) regulates Vibrio cholerae biofilm formation. Mol. Microbiol. 53, 857-869. doi: 10.1111/j.1365-2958.2004.04155.x

Tischler, A. D., and Camilli, A. (2005). Cyclic diguanylate regulates Vibrio cholerae virulence gene expression. Infect. Immun. 73, 5873-5882. doi: 10.1128/IAI.73.9.5873-5882.2005

Utada, A. S., Bennett, R. R., Fong, J. C. N., Gibiansky, M. L., Yildiz, F. H., Golestanian, R., et al. (2014). Vibrio cholerae use pili and flagella synergistically to effect motility switching and conditional surface attachment. Nat. Commun. 5:4913. doi: 10.1038/ncomms5913

Wang, Y.-C., Chin, K.-H., Tu, Z.-L., He, J., Jones, C. J., Zamorano-Sánchez, D., et al. (2016). Nucleotide binding by the widespread high-affinity cyclic diGMP receptor MshEN domain. Nat. Commun. 7:12481. doi: 10.1038/ncomms 12481

Waters, C. M., Lu, W., Rabinowitz, J. D., and Bassler, B. L. (2008). Quorum sensing controls biofilm formation in Vibrio cholerae through modulation of cyclic di-GMP levels and repression of vpsT. J. Bacteriol. 190, 2527-2536. doi: 10.1128/JB.01756-07

Watnick, P. I., Fullner, K. J., and Kolter, R. (1999). A role for the mannose-sensitive hemagglutinin in biofilm formation by Vibrio cholerae El Tor. J. Bacteriol. 181, 3606-3609. doi: 10.1128/JB.181.11.3606-3609.1999

Watnick, P. I., and Kolter, R. (2000). Biofilm, city of microbes. J. Bacteriol. 182, 2675-2679. doi: 10.1128/JB.182.10.2675-2679.2000

Wong, G. C. L. (2016). Three-dimensional architecture of Vibrio cholerae biofilms. Proc. Natl. Acad. Sci. U.S.A. 113, 3711-3713. doi: 10.1073/pnas.1603016113

Xu, M., Wang, Y. Z., Yang, X. A., Jiang, T., and Xie, W. (2017). Structural studies of the periplasmic portion of the diguanylate cyclase $\mathrm{CdgH}$ from Vibrio cholerae. Sci. Rep. 7:1861. doi: 10.1038/s41598-017-01989-6

$\mathrm{Xu}$, Q., Dziejman, M., and Mekalanos, J. J. (2003). Determination of the transcriptome of Vibrio cholerae during intraintestinal growth and 
midexponential phase in vitro. Proc. Natl. Acad. Sci. U.S.A. 100, 1286-1291. doi: 10.1073/pnas.0337479100

Yildiz, F. H., Liu, X. S., Heydorn, A., and Schoolnik, G. K. (2004). Molecular analysis of rugosity in a Vibrio cholerae O1 El Tor phase variant. Mol. Microbiol. 53, 497-515. doi: 10.1111/j.1365-2958.2004. 04154.x

Yildiz, F. H., and Schoolnik, G. K. (1999). Vibrio cholerae O1 El Tor: identification of a gene cluster required for the rugose colony type, exopolysaccharide production, chlorine resistance, and biofilm formation. Proc. Natl. Acad. Sci. U.S.A. 96, 4028-4033. doi: 10.1073/pnas.96.7.4028

Zamorano-Sánchez, D., Xian, W., Lee, C. K., Salinas, M., Thongsomboon, W., Cegelski, L., et al. (2019). Functional specialization in Vibrio cholerae diguanylate cyclases: distinct modes of motility suppression and c-di-GMP production. mBio 10, e00670-e00619. doi: 10.1128/mBio. 00670-19
Zhao-Xun, L. (2015). The expanding roles of c-di-GMP in the biosynthesis of exopolysaccharides and secondary metabolites. Nat. Prod. Rep. 32, 663-683. doi: $10.1039 / \mathrm{C} 4 \mathrm{NP} 00086 \mathrm{~B}$

Conflict of Interest: The authors declare that the research was conducted in the absence of any commercial or financial relationships that could be construed as a potential conflict of interest.

Copyright $\odot 2020$ Biswas, Chouhan and Bandekar. This is an open-access article distributed under the terms of the Creative Commons Attribution License (CC BY). The use, distribution or reproduction in other forums is permitted, provided the original author(s) and the copyright owner(s) are credited and that the original publication in this journal is cited, in accordance with accepted academic practice. No use, distribution or reproduction is permitted which does not comply with these terms. 